\title{
The chromosome-level reference genome of Coptis chinensis provides insights into genomic evolution and berberine biosynthesis
}

Da-xia Chen 1,2,3, Yuan Pan ${ }^{1,2,3}$, Yu Wang ${ }^{1,2,3}$, Yan-Ze Cui ${ }^{4}$, Ying-Jun Zhang ${ }^{4}$, Rang-yu Mo ${ }^{1,2,3}$, Xiao-li Wu ${ }^{1,2,3}$, Jun Tan ${ }^{1,2,3}$, Jian Zhang ${ }^{1,2,3}$, Lian-an Guo ${ }^{1,2,3}$, Xiao Zhao ${ }^{1,2,3}$, Wenkai Jiang ${ }^{4}$, Tian-lin Sun ${ }^{4}$, Xiao-Di Hu ${ }^{4}$ and Long-yun Li $i^{1,2,3}$

\begin{abstract}
Coptis chinensis Franch, a perennial herb, is mainly distributed in southeastern China. The rhizome of $C$. chinensis has been used as a traditional medicine for more than 2000 years in China and many other Asian countries. The pharmacological activities of $C$. chinensis have been validated by research. Here, we present a de novo high-quality genome of C. chinensis with a chromosome-level genome of $\sim 958.20 \mathrm{Mb}$, a contig N50 of $1.58 \mathrm{Mb}$, and a scaffold N50 of $4.53 \mathrm{Mb}$. We found that the relatively large genome size of $C$. chinensis was caused by the amplification of long terminal repeat (LTR) retrotransposons. In addition, a whole-genome duplication event in ancestral Ranunculales was discovered. Comparative genomic analysis revealed that the tyrosine decarboxylase (TYDC) and (S)-norcoclaurine synthase (NCS) genes were expanded and that the aspartate aminotransferase gene (ASP5) was positively selected in the berberine metabolic pathway. Expression level and HPLC analyses showed that the berberine content was highest in the roots of $C$. chinensis in the third and fourth years. The chromosome-level reference genome of $C$. chinensis provides important genomic data for molecular-assisted breeding and active ingredient biosynthesis.
\end{abstract}

\section{Introduction}

Coptis chinensis Franch or Rhizoma Coptis $(2 n=2 \mathrm{X}=$ 18, Ranunculaceae) is a well-known medicinal plant that is mainly cultivated in Chongqing, Hubei, Hunan, Shanxi, and Guizhou, China. C. chinensis prefers to grow in 1200-1800 m high mountains and damp, shady and cold environments. In China, C. chinensis is called Huanglian, Weilian, Chuanlian or Jizhualian, and its rhizome has been used as a traditional Chinese medicine for more than 2000 years $^{1,2}$. The search for plant-derived alkaloids beneficial to human health has received widespread attention. The pharmacological activities of C. chinensis

Correspondence: Xiao-Di Hu (huxiaodi@novogene.com) or Longyun Li (lilongyun8@163.com)

${ }^{1}$ Chongqing Academy of Chinese Materia Medica, 400065 Chongqing, China

${ }^{2}$ Chongqing Engineering Research Center for Fine Variety Breeding

Techniques of Chinese Materia Medica, 400065 Chongqing, China

Full list of author information is available at the end of the article

These authors contributed equally: Da-xia Chen, Yuan Pan, Yu Wang, Yan-

Ze Cui rhizomes have been proven to play an important role in preventing or attenuating the development or progression of diseases ${ }^{1,3-5}$. Many studies have demonstrated the pharmacological activities of Huanglian rhizome preparations, especially the activity of novel alkaloids ${ }^{1,3-5}$. The bioactivities of $C$. chinensis alkaloids include broadspectrum antimicrobial ${ }^{5}$, anticancer ${ }^{6}$, antioxidant ${ }^{7,8}$, antidiabetic $^{9,10}$, attenuation depressive-like ${ }^{11}$, antiadipogenic $^{12}$, and anti-inflammatory ${ }^{13,14}$ properties. Generally, C. chinensis mainly consists of six alkaloids, i.e., berberine, coptisine, palmatine, jatrorrhizine, epiberberine, and columbamine ${ }^{15}$. Among them, berberine ( $\left.7 \%\right)$, the dominant alkaloid, is known to have multiple beneficial physiological effects ${ }^{16}$. Despite the beneficial properties and commercial interest in $C$. chinensis, the distribution and number of wild $C$. chinensis are very limited.

At present, C. chinensis mostly originates from cultivation and is mainly produced in the Shizhu Tujia 
Table 1 Statistics for assembly and annotation of the Coptis chinensis genome.

\begin{tabular}{lll}
\hline Characteristic & Number & Size \\
\hline Assembly & & \\
Estimated genome size & 425 & $1046.91 \mathrm{Mb}$ \\
Scaffolds & 63 & $958.20 \mathrm{Mb}$ \\
N50 of scaffolds & & $4.53 \mathrm{Mb}$ \\
Longest scaffolds & 940 & $19.44 \mathrm{Mb}$ \\
Contigs & 187 & $955.01 \mathrm{Mb}$ \\
N50 of contigs & & $1.58 \mathrm{Mb}$ \\
Longest contigs & & $8.97 \mathrm{Mb}$ \\
Annotation & $62.23 \%$ & $596.28 \mathrm{Mb}$ \\
Repetitive sequences & $60.75 \%$ & $582.07 \mathrm{Mb}$ \\
Transposable element & 34,109 & \\
Protein-coding genes & & $3846.49 \mathrm{bp}$ \\
Mean transcript length & & $1031.05 \mathrm{bp}$ \\
Mean coding sequence length & 5424 & $615,322 \mathrm{bp}$ \\
Noncoding RNAs & &
\end{tabular}

Autunomous County of Chongqing. C. chinensis has several special characteristics, including seed propagation, introduction to different regions, facultative outcrossing, self-flowering, and cross-pollination coexistence. Therefore, the cultivated populations of $C$. chinensis contain rich genetic resources that are diverse and highly heterozygous, and it is thus difficult to perform plant purification and breed selection.

To date, no species in the Coptis genus has a fully sequenced genome. High-quality genomes enable comparative analyses of genome architecture and the evolution of key traits for seed plants ${ }^{17-19}$. Despite the importance of $C$. chinensis as an ideal traditional medicine, genetic research on Huanglian is far from well developed, with no high-quality reference genomes. Here, we present the first high-quality genome assembly of $C$. chinensis using combined approaches, including PacBio (single-molecule Pacific Biosciences), Illumina HiSeq X, and $\mathrm{Hi}-\mathrm{C}$ (genome-wide chromosome conformation capture) technology. This assembly contains 9 chromosomes $(\mathrm{N} 50=4.53 \mathrm{Mb})$ totaling $958.20 \mathrm{Mb}$. The highquality genomes of $C$. chinensis will allow us to uncover the genetic mechanisms behind special features such as the biosynthesis of alkaloids.

\section{Results}

\section{Genome assembly and feature annotation}

The genome size was estimated $\sim 1046.91 \mathrm{Mb}$, the heterozygosity proportion was 0.77 , and the repeat ratio was
$73.96 \%$ based on k-mer analysis (Table S1, Fig. S1). A total of $\sim 136.69 \mathrm{~Gb}$ of raw reads with an inserted $350 \mathrm{bp}$ library $(\sim 130.57 \mathrm{X}), \quad 118.36 \mathrm{~Gb}$ of PacBio data ( 113.06X), $130.73 \mathrm{~Gb}$ of $10 \mathrm{X}$ genomics data $(\sim 124.87 \mathrm{X})$, and 95.99 $\mathrm{Gb}$ of clean $\mathrm{Hi}-\mathrm{C}$ reads $(\sim 91.69 \mathrm{X})$ were used for assembly (Table S2), yielding a draft genome of $958.20 \mathrm{Mb}$ with a contig $\mathrm{N} 50$ of $1.58 \mathrm{Mb}$ and a scaffold N50 of $4.53 \mathrm{Mb}$, which is close to the estimates based on k-mer analysis (Table S3). Approximately $95.69 \%$ of the assembled scaffolds were anchored to nine pseudochromosomes based on Hi-C data (Fig. S2, Tables 1, S4).

The mapping rate reached $96.79 \%$ when Illumina reads were aligned with our assembled genome, and the completeness of our genome assembly was evaluated by CEGMA $^{20}$ and BUSCO assessment. The results showed that a total of $236(95.16 \%)$ of 248 core eukaryotic genes (CEGs) and 1523 (94.3\%) complete gene models among 1614 conserved genes from BUSCO assessment were identified (Tables S5, S6, S7), thus supporting that our genome assembly has a high quality and completeness. Additionally, LTR annotation was performed to estimate the assembly genome quality. Compared with Aquilegia oxysepala $(16.7)^{21}$ and Aquilegia coerulea $(12.6)^{22}$ in the family Ranunculaceae, a high LAI score of 17.86 showed that the assembly of $C$. chinensis yielded high sequence continuity and met the reference quality ${ }^{23}$. Overall, the assembled $C$. chinensis genome contains $595.32 \mathrm{Mb}$ $(62.13 \%)$ of repetitive sequences, most of which are transposable elements (581.03 Mb; 60.63\%). Long terminal repeat (LTR) retrotransposons represent $49.84 \%$ of the genome, the largest percentage of repetitive elements (Tables S8, S9, Figs. 1, S3).

We identified 34,109 protein-coding genes (PCGs) in $C$. chinensis with an average of 4.70 exons per gene and an average coding sequence (CDS) length of $1031.26 \mathrm{bp}$. Of these, 33,898 (99.40\%) could be functionally annotated in at least one database, i.e., NR, SwissProt, KEGG and InterPro, by homology. A total of 25,147 (73.70\%) genes contained Pfam domains, and 30,773 (90.10\%) genes were assigned GO terms. In addition, noncoding RNAs were also annotated in the $C$. chinensis genome, yielding 1,050 miRNAs, 996 tRNAs, 2,112 rRNAs, and 1265 snRNAs with average lengths of 131.56, 76.38, 128.96, and $116.68 \mathrm{bp}$, respectively (Tables $1, \mathrm{~S} 10, \mathrm{~S} 11, \mathrm{~S} 12$ ).

\section{Comparative genomic analysis and whole-genome duplication (WGD)}

To better understand the evolution of C. chinensis, we compared the sequence similarity of $C$. chinensis and 8 other species (Aquilegia coerulea, Arabidopsis thaliana, Artemisia annua, Glycyrrhiza uralensis, Macleaya cordata, Nelumbo nucifera, Salvia miltiorrhiza, and Oryza sativa) using OrthoMCL ${ }^{24}$. A total of 26,614 gene families were shared among $C$. chinensis and the eight other 


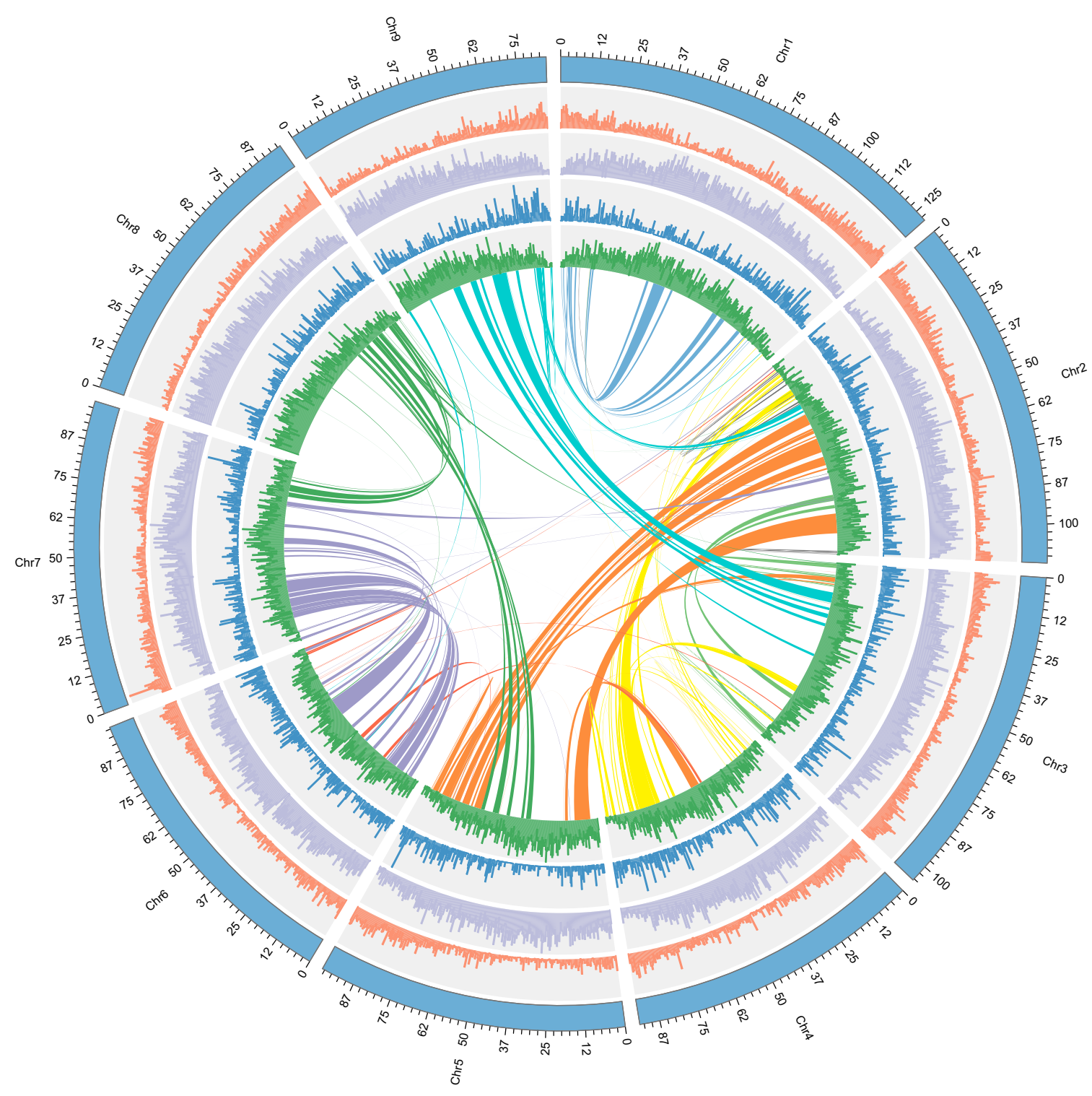

Fig. 1 Genome features across 9 chromosomes. The outermost circle (blue) represents each chromosome of the genome. The bar charts of the second to fifth circles suggest gene density, LTR density, Copia and Gypsy density, respectively. The inner circular representation shows interchromosomal synteny. All sections were drawn based on window size $=300 \mathrm{~kb}$ and chromosome units $=500 \mathrm{~kb}$

species, and 817 common single-copy orthologous gene families were identified according to sequence similarity. By comparing the gene families of Coptis chinensis, Aquilegia coerulea, Arabidopsis thaliana, Macleaya cordata, and Nelumbo nucifera, 1934 Coptis chinensis-specific gene families consisting of 5352 genes were detected (Fig. 2a). These genes were used to perform Kyoto Encyclopedia of Genes and Genomes (KEGG) functional annotation, and nine KEGG pathways were significantly enriched (cutoff $<0.05$ ) (Table S13). The enriched phenylalanine, tyrosine, and tryptophan biosynthesis (map00400) could provide substrates for isoquinoline alkaloid biosynthesis, which probably has a potential contribution to the synthesis of benzylisoquinoline (BIAs).

A total of 817 single-copy orthologous gene families were used to perform phylogenetic analysis to confirm the relationship between Coptis chinensis and other species. According to phylogenetic tree results, $C$. chinensis, $A$. coerulea, $M$. cordata, and $N$. nucifera were clustered in the same branch, which belongs to the basal eudicot clade, 
a

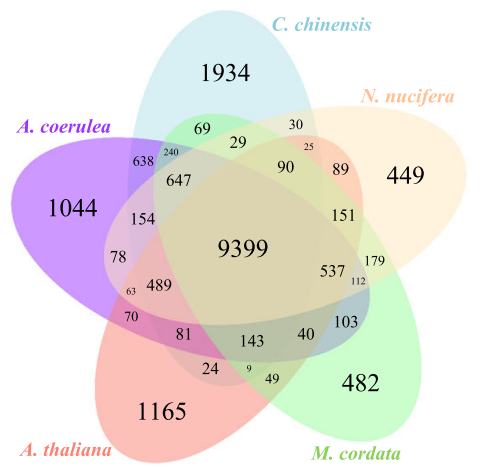

c

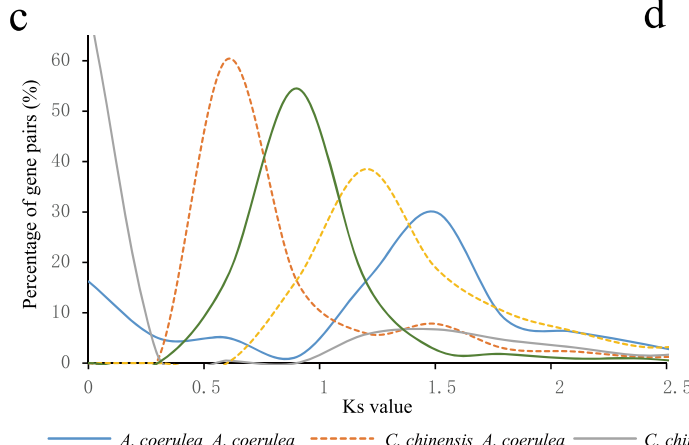

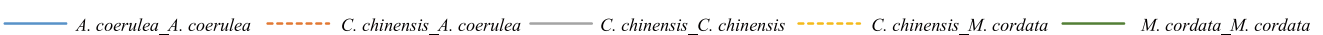

$\mathrm{b}$

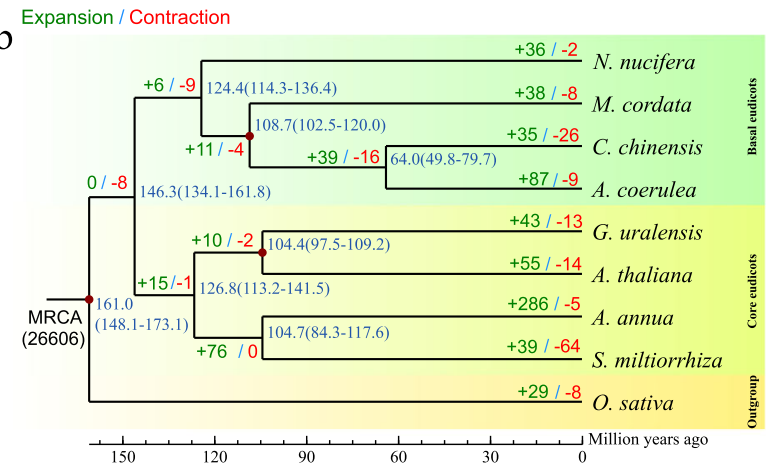

d

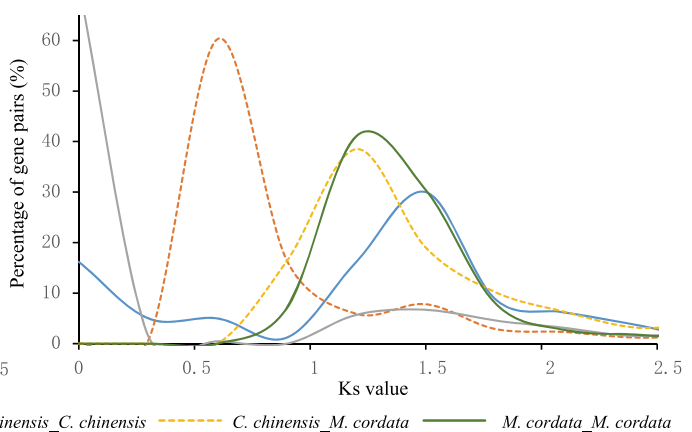

Fig. 2 Comparison of gene families. a Venn diagrams displaying the number of gene families shared among five species. $\mathbf{b}$ Phylogenetic tree constructed by 817 single-copy genes. The divergence time is given in millions of years in blue. The gene families that expanded and contracted are given in green and red, respectively. c, d Percentage distribution of Ks for orthologous and paralogous gene pairs. The $x$-axis denotes the Ks value. The $y$-axis denotes the percentage of gene pairs. Rate distributions were not corrected (c); Macleaya cordata rates were corrected for Coptis chinensis (d)

and the result is consistent with their previously reported phylogenetic relationship. Further divergence time analysis showed that the Proteales order (N. nucifera) separated from the Ranunculales order (A. coerulea, $C$. chinensis, and M. cordata) 124.4 (114.3-136.4) Mya ago, and $C$. chinensis separated from the same family species, A. coerulea, $\sim 64.0$ (49.8-79.7) Mya (Fig. 2b). We also detected the expanded or contracted gene families using CAFÉ (version 1.6). In total, 26,606 gene families were inferred in the most recent common ancestor (MRCA) of the nine species. Compared with the MRCA of C. chinensis and $A$. coerulea, 35 significantly expanded and 26 significantly contracted gene families were exhibited in C. chinensis (Fig. 2b). Genes in the 35 significantly expanded families were used to perform functional annotation based on the KEGG database, and ten KEGG pathways were significantly enriched (cutoff $<0.05$ ) (Table S14). The enriched isoquinoline alkaloid biosynthesis (map00950) potentially demonstrates the genomic basis for the high BIA content in C. chinensis. All five genes enriched in this pathway belong to the tyrosine decarboxylase (TYDC) family, which is involved in the synthesis of premises (dopamine and 4-hydroxyphenylacetaldehyde) of BIAs. Thirty positively selected genes were identified in C. chinensis. According to the KEGG functional classification, isoquinoline alkaloid biosynthesis (map00950) was also enriched, and the enriched gene aspartate aminotransferase (ASP5) was also involved in the synthesis of premises (4-hydroxyphenylacetaldehyde) of BIAs (Table S15).

By calculating the Ks values of duplicate gene pairs, we observed a peak at Ks values of 1.5 (Fig. 2c, d), indicating that $C$. chinensis likely underwent a WGD event and that the peak of $\sim 0$ values of $C$. chinensis may be caused by tandem repeats. The peak value of orthologs between $C$. chinensis and A. coerulea $(\mathrm{Ks}=0.6)$ was lower than the value of $\mathrm{Ks}=1.2$ between $C$. chinensis and $M$. cordata (Fig. 2c), implying that the speciation between $C$. chinensis and $A$. coerulea occurred later, and this result corresponds to the phylogenetic relationship. According to the distribution of Ks values, the WGD event of $M$. cordata occurred after speciation with $C$. chinensis, which suggests that $M$. cordata would share another WGD event with $C$. chinensis (Fig. 2c). However, there is no evidence to support that $M$. cordata had additional WGD events ${ }^{25}$. Such a result could be due to the relatively slow evolution of $M$. cordata. To confirm this, we determined Ks rates between $C$. chinensis and $N$. nucifera, finding that the 
mean Ks was larger than that between $M$. cordata and $N$. nucifera. Then, we adjusted the Ks distributions of $M$. cordata according to the ratio of the Ks value of the orthologous gene pairs between $C$. chinensis- $N$. nucifera and $M$. cordata- $N$. nucifera according to the method of Paterson et al. ${ }^{26}$ and Pei et al. $^{22}$. The adjusted result showed that $C$. chinensis, A. coerulea and M. cordata occupied almost the same position, strongly implying that they shared a common WGD event (Fig. 2d).

\section{LTR retrotransposon expansion leads to a large genome} size

Compared with the genomes of two closely related species, $M$. cordata $(\sim 378 \mathrm{Mb})^{25}$ and A. coerulea $(\sim 307 \mathrm{Mb})^{27}$, the genome size of C. chinensis $(\sim 958 \mathrm{Mb})$ is much larger. Transposable elements, especially LTRs, are essential for the formation of genome structure. The evolution of LTRs was investigated to explore the potential contribution to the enlargement of the C. chinensis genome. C. chinensis harbors the highest content of LTRs $(\sim 461.6 \mathrm{Mb})$, compared with the other two closely related species in Ranunculales: $M$. cordata $(\sim 104.8 \mathrm{Mb})^{25}$ and A. coerulea $(\sim 100.3 \mathrm{Mb})^{27}$. To trace the history of the expanded LTRs in C. chinensis, we identified LTRs and estimated insertion times of all intact LTRs in these species. A total of 23,433, 5231, and 6370 intact LTRs were identified in C. chinensis, M. cordata, and A. coerulea, respectively. In the genomes of $M$. cordata, no significant proliferation of LTRs was observed. In the genome of $A$. coerulea, there were obviously more LTR insertions than in $M$. cordata in the last 2 Mya. LTRs continuously and substantially accumulated only in the genome of C. chinensis in the last $10 \mathrm{Mya}$, which showed a relatively longer expansion period than in the other two species (Fig. S4).
Approximately $81.67 \%$ of the intact LTRs in the $C$. chinensis genome had at least one protein domain, of which the vast majority were Ty1/copia and Ty3/gypsy, accounting for $19.40 \%$ and $80.53 \%$, respectively, and their corresponding total lengths were 23.96 and $187.96 \mathrm{M}$. The proportion of Ty3/gypsy elements in the $C$. chinensis genome was significantly higher than that in $M$. cordata (47.41\%) and A. coerulea (52.42\%). In addition, the total length of Ty3/gypsy elements in the C. chinensis genome was 12.51 times longer than that in M. cordata and 8.77 times longer than that in A. coerulea. Ty1/copia elements also expanded in C. chinensis, with total lengths 1.83 and 1.73 times those of $M$. cordata and A. coerulea, respectively (Table S16). The evolutionary relationships of individual Ty1/copia and Ty3/gypsy LTR superfamilies were studied in these three species. All Ty3/gypsy elements from these three species were grouped into six major evolutionary clades, i.e., Angela, Ale, Bianca, Ivana, Maximus, and TAR (Table S17). The Ty3/gypsy elements of $M$. cordata and A. coerulea were separated into six major evolutionary lineages: Tekay, Galadriel, CRM, Reina, Athila, and Tat. However, the Ty1/copia elements of $C$. chinensis were assigned to five distinct lineages, of which the Galadriel lineage was not found (Table S18). To understand the amplification of individual lineages, we calculated the copy numbers and constructed phylogenetic trees of Ty1/copia and Ty3/gypsy. There was no significant expansion of Ty1/copia and Ty3/gypsy in $M$. cordata. In A. coerulea, the Tat of Ty3/gypsy showed significant expansion, which accounted for $78.46 \%$ of the total (Table S18, Fig. 3b), which was consistent with more LTR insertions in A. coerulea over the last 2 Mya. More LTR insertions resulted in a higher proportion of LTRs in A. coerulea (32.67\%) than in M. cordata (27.72\%). In C. chinensis, the significantly expanded lineage of the Ty1/
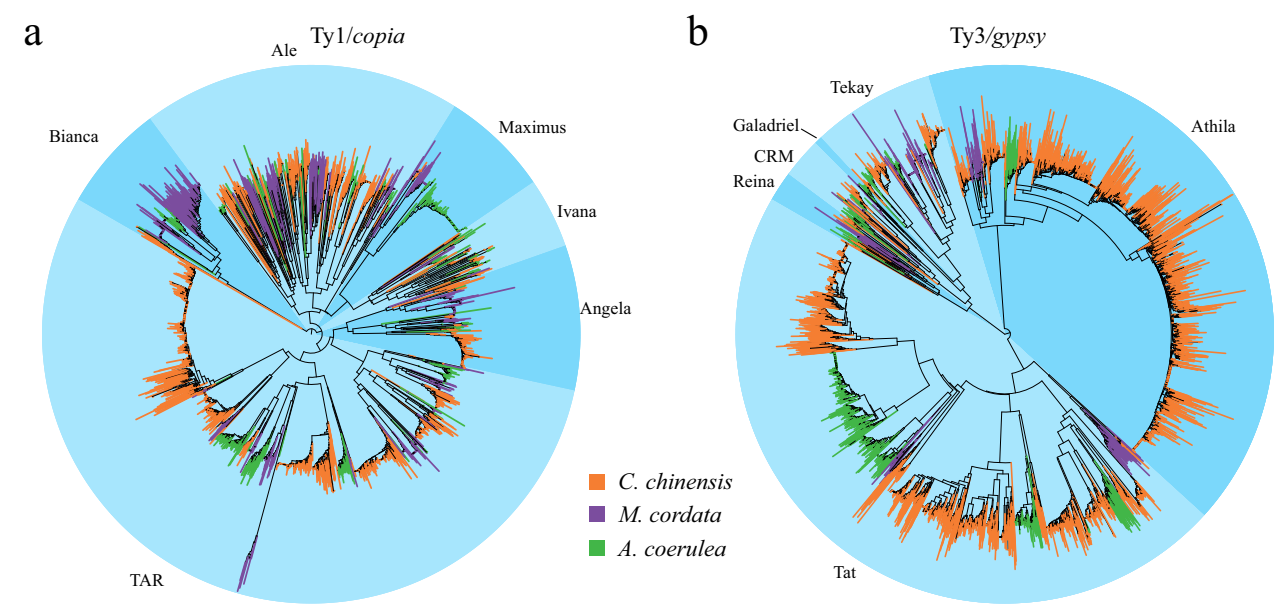

Fig. 3 Phylogenetic analysis of LTRs in the C. chinensis genome. The neighbor-joining phylogenetic trees based on Ty1/copia (a) and Ty3/gypsy (b). Major lineages are named, and the proportion is indicated 
copia superfamily was TAR, which accounted for $69.09 \%$ (Table S17, Fig. 3a), and the Athila and Tat of Ty3/gypsy also showed significant expansion, which accounted for $40.17 \%$ and $53.02 \%$, respectively (Table S17, Fig. $3 b$ ). The three significantly expanded lineages may be drivers of the expanded genome of C. chinensis (Tables S16-18, Fig. 3).

\section{Benzylisoquinoline alkaloid biosynthesis-associated genes}

In total, 63 candidate genes were identified based on 14 previously reported genes involved in BIA biosynthesis, i.e., tyrosine decarboxylase (TYDC), tyrosine/tyramine 3hydroxylase/tyrosine 3-monooxygenase (3OHase), Ltyrosine aminotransferase (TyrAT), (S)-norcoclaurine synthase (NCS), (RS)-norcoclaurine 6-O-methyltransferase (6OMT), (S)-coclaurine $\mathrm{N}$-methyltransferase (CNMT), (S)$\mathrm{N}$-methylcoclaurine $\quad 3^{\prime}$-hydroxylase/N-methylcoclaurine $3^{\prime}$-monooxygenase $(\mathrm{NMCH}), \quad 3^{\prime}$-hydroxy- $\mathrm{N}$-methyl-(S)coclaurine $4^{\prime}$-O-methyltransferase $\left(4^{\prime} O M T\right)$, berberine bridge enzyme (reticuline oxidase) $(B B E)$, (S)-scoulerine 9O-methyltransferase (SOMT), (S)-canadine synthase

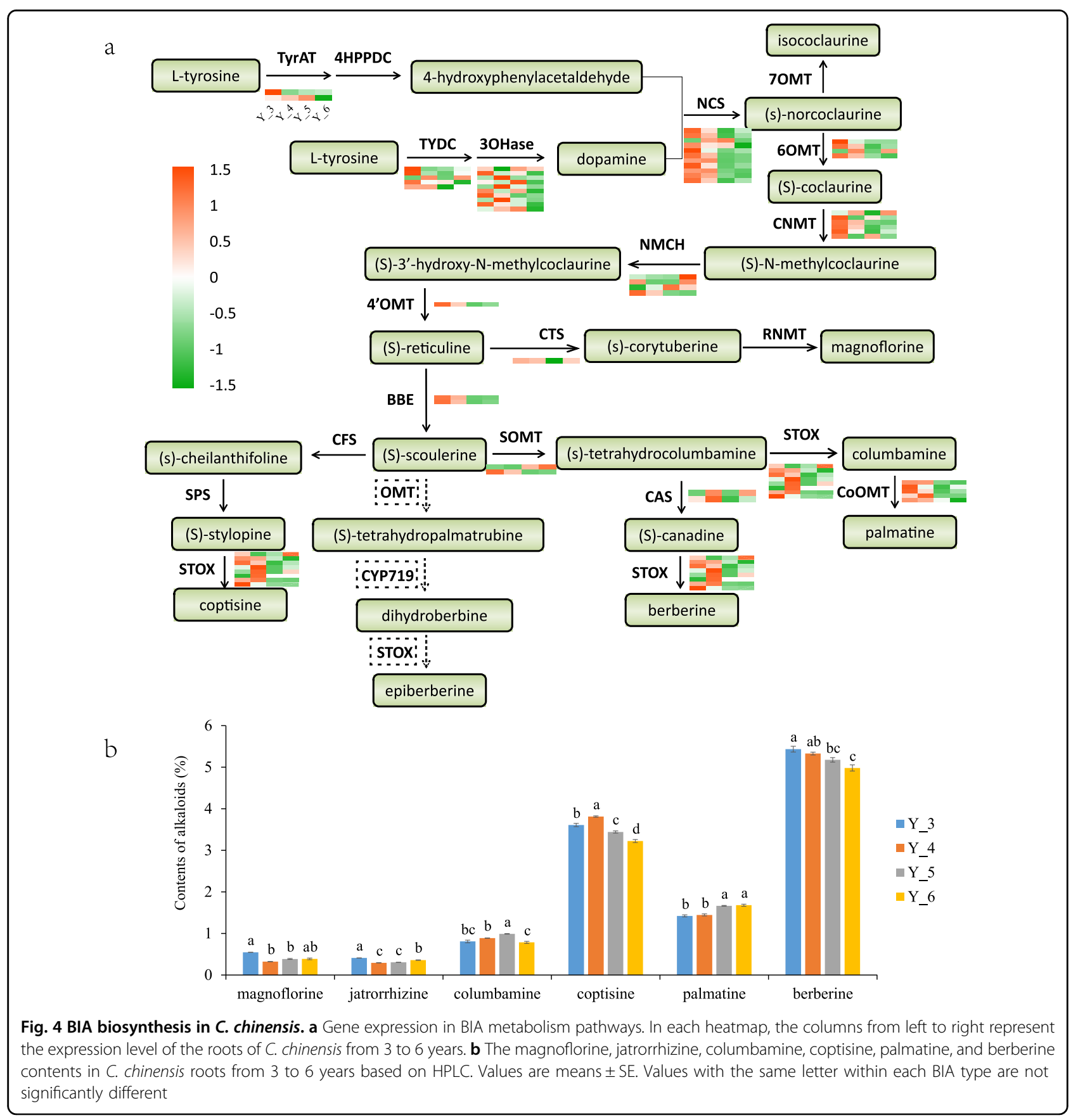


(CAS), (S)-tetrahydroprotoberberine oxidase (STOX), (S)-corytuberine synthase (CTS), columbamine Omethyltransferase (CoOMT), and reticuline $\mathrm{N}$ methyltransferase $(R N M T){ }^{28}$. To clarify the specific evolution of genes involved in BIA synthesis in Ranunculales, gene orthologs were also identified in $M$. cordata, $A$. coerulea and $A$. thaliana. (S)-Reticuline is an intermediate required for the synthesis of many BIAs. Compared with Arabidopsis thaliana, four genes (NCS, 6OMT, NMCH, and $4^{\prime} O M T$ ) involved in the pathways from L-tyrosine to (s)-reticuline could only be found in three Ranunculales plants (Table S19), and another key gene, CNMT, was also significantly expanded in Ranunculales plants. Comparative analysis with Aquilegia coerulea and Macleaya cordata showed that the key gene CoOMT responsible for catalyzing columbamine into palmatine expanded in $C$. chinensis, and the key gene NCS involved in the synthesis of (S)-norcoclaurine, a precursor to BIAs, also expanded in C. chinensis (Table S19).

To better understand the molecular mechanisms and regulatory processes of BIAs, we investigated the expression patterns of genes potentially related to BIA biosynthesis from five tissues (flowers, seeds, roots, fibrous roots, and leaves) at the same stage and roots from 3 to 6 years. According to previous research, berberine, coptisine and jatrorrhizine were significantly more abundant in the roots than in the rhizomes and leaves of $C$. chinensis ${ }^{28}$. In our study, the expression level analysis of different tissues showed that the genes STOX and CoOMT, acting on the final catalytic for the synthesis of predominant BIAs (berberine, coptisine, and palmatine), were significantly overexpressed in fibrous roots and roots compared with other tissues. In addition, the expression levels of the key genes $B B E$ and $4^{\prime} O M T$ were also higher in roots. Many genes, those with multiple copies, exhibited an obvious divergence in expression pattern among copies from different tissues, such as NCS, NMCH, and CNMT (Fig. S5). As the BIA content was the highest in the roots of $C$. chinensis, we further analyzed the expression levels of roots in different years. Most of the candidate genes related to BIA synthesis were most highly expressed in the third year, followed by the fourth year, and were significantly decreased in the fifth and sixth years (Figs. S6, 4a), which suggests that the key period for BIA synthesis in C. chinensis is the third and fourth years.

To verify that the candidate genes were closely related to the content of BIAs, high-performance liquid chromatography (HPLC) analysis was used to detect the presence of magnoflorine, jatrorrhizine, columbamine, berberine, coptisine, and palmatine in the roots of $C$. chinensis from 3 to 6 years. In general, the highest proportion of BIAs in the roots of C. chinensis was berberine, followed by coptisine and palmatine. The content of predominant BIAs (berberine and coptisine) in $C$. chinensis reached the highest level in the fourth year and then decreased, which indicates that BIAs in C. chinensis accumulated until the fourth year (Fig. 4b). Correlation coefficient analysis of gene expression patterns and various alkaloid contents was used to further validate candidate genes. The results showed that most of the key genes were highly correlated with the predominant components (berberine and palmatine), with $R^{2}>0.8$, such as NCS, 6OMT, CNMT, BBE, and STOX (Fig. S7). Coptisine is also the predominant component of BIAs in C. chinensis; however, CFS and SPS genes related to the synthesis of coptisine have not been identified, which may be because other enzymes of the CYP719 family have similar functions or there are other bypass pathways for the synthesis of (S)-stylopine. In addition, recently duplicated gene blocks $(\mathrm{Ks}<0.3)$ were used to investigate the effects on BIA synthesis in C. chinensis. We found that $3 O H a s e$ 6OMT and CNMT were in the recently duplicated blocks (Fig. S8).

\section{Discussion}

A high-quality, chromosome-level genome provides a key resource to study how biosynthetic pathways evolved $^{21}$. To date, only genomes of Aquilegia oxysepala ${ }^{29}$ and Aquilegia coerulea ${ }^{27}$ from the family Ranunculaceae have been reported. In our study, we obtained a highquality genome of cultivated $C$. chinensis using a combined approach. The final chromosome-level genome assembly is $\sim 958.20 \mathrm{Mb}$, which is close to the assessed genome size $(1.06 \mathrm{~Gb})$, and similar to the previous version of the C. chinensis genome ${ }^{30}$. The assembled genome has a high quality and completeness, with a contig N50 of $1.58 \mathrm{Mb}$ and a scaffold N50 of $4.53 \mathrm{Mb}$; in total, 34,109 PCGs were predicted. This is the first chromosome-level reference genome of $C$. chinensis, which provides important genomic data for $C$. chinensis studies.

The first genome in the Coptis genus facilitates further research on fundamental comparative and genome evolution. Among Coptis-expanded and positive genes, isoquinoline alkaloid biosynthesis activity is one of the more highly enriched KEGG pathways, including the genes (TyrAT and TYDC) related to the conversion from Ltyrosine to 4-hydroxyphenylacetaldehyde and dopamine, suggesting that they are part of the genomic basis for the high content of isoquinoline, including berberine, in $C$. chinensis. Comparison of the C. chinensis, A. coerulea and $M$. cordata genomes supports the hypothesis that basal eudicot plants lack the paleohexaploidy event and that the ancestor of Ranunculales shared a common WGD ${ }^{25,29}$ (Fig. 2d). The distribution of Ks values of duplicated gene pairs in corresponding blocks suggests that the divergence of $C$. chinensis $-A$. coerulea occurred later than that of $C$. chinensis $-M$. cordata (Fig. 2d). C. chinensis possesses a significantly enlarged genome compared with closely 
related species in the Ranunculaceae family. A positive correlation between the content of LTRs and genome size was previously reported for maize ${ }^{31}$, pineapple $^{32}$, and tea tree $^{33}$. In our study, the repeat-sequence contents of TAR, Athila and Tat of LTR lineages were drivers of the enlarged C. chinensis genome.

Furthermore, we also detected genes related to BIAs in the $C$. chinensis genome, and the contents of BIAs were detected by HPLC. The biosynthesis of BIAs starts with the transformation from L-tyrosine to 4hydroxyphenylacetaldehyde and dopamine, by which (S)-norcoclaurine is synthesized under the catalysis of NCS. Three methyltransferases (6OMT, CNMT, and $4^{\prime}$ $O M T)$ and one cytochrome $\mathrm{P} 450(\mathrm{NMCH})$ are associated with the transformation from (S)-norcoclaurine to (S)reticuline, which is necessary for the synthesis of multiple BIAs. The NCS genes involved in this process are significantly expanded in Coptis chinensis, which may provide sufficient substrates for downstream synthesis. Some key genes related to BIA synthesis, such as STOX, CoOMT, $B B E$, and $4^{\prime} O M T$, have high expression levels in roots and fibrous roots. This suggests that roots are the main organ for the synthesis of BIAs, which is consistent with a previous study ${ }^{28}$. Consistent with the HPLC analysis results, most candidate genes related to BIA synthesis were highly expressed in the third and fourth years, indicating that these candidate genes contributed to the synthesis of BIAs and provide basic data for the selection of the best medicinal period for $C$. chinensis. Three key genes for the synthesis of the intermediate (S)-reticuline (3OHase 6OMT and CNMT) were recently duplicated (Fig. S8), suggesting that recent duplications may have a positive effect on the biosynthesis of BIAs in Coptis chinensis.

For Coptis chinensis, an important medicinal plant, the reported chromosome-level genome lays a foundation for the analysis of comparative genomes and will contribute to molecular breeding, as well as the elucidation of BIA metabolic pathways.

\section{Materials and methods Plant materials}

Diploid C. chinensis was originally collected from the Chongqing Academy of Chinese Materia Medica, Chongqing, China $\left(29.99^{\circ} \mathrm{N}, 108.44^{\circ} \mathrm{E}, 1667 \mathrm{~m}\right.$ asl). Young leaves in a slowly growing period from a 5-year-old individual were used for genome sequencing and assembly.

\section{Library construction and genome sequencing}

Genomic DNA was extracted from fresh leaf tissues of C. chinensis using the plant Genomic DNA Kit (Tiangen, Beijing, PR China) according to the manufacturer's instructions. Libraries of $350 \mathrm{bp}$ were constructed and sequenced using the Illumina HiSeq platform (Illumina, San Diego, CA).

Low-quality reads and sequences with adapters were removed from the raw data. The following reads were removed from the raw data:

(1) Reads containing adapters.

(2) Paired reads containing unidentified nucleotides $(\mathrm{N})$ $>10 \%$ of the total bases of single reads.

(3) Paired reads containing more than $20 \%$ low-quality bases (Phred $<5)$ of the total length of single reads.

Libraries of $20 \mathrm{~kb}$ for single-molecule real time require at least $10 \mu \mathrm{g}$ of sheared DNA. The libraries were sequenced using the PacBio Sequel platform. Hi-C libraries were also enriched, sheared, and sequenced using Illumina HiSeq. Five tissues (leaf, seed, flower, root, and fibrous root) from the same individual were collected for RNA sequencing.

\section{De novo genome assembly and genome quality assessment}

$\mathrm{K}$-mer frequency analysis was performed ${ }^{34}$ to estimate the $C$. chinensis genome size, heterozygosity and repeat content. A 17-mer frequency was generated from highquality 250 and 450 bp paired-end reads. The distribution of 17-mers following a Poisson's distribution can reflect the characteristics of the genome. The long subreads generated by PacBio were assembled using the following steps. First, error correction of the raw subreads was performed to obtain preassembled reads, which generally have a very high accuracy (up to $99.999 \%$ ). Then, Falcon ${ }^{35}$ software was applied to assemble the preassembled reads using the overlap-layout-consensus (DBG2OLC) method. The production of primary contigs (p-contigs) was performed using FALCON-Unzip ${ }^{35}$, and then Quiver ${ }^{36}$ was used for polishing. Finally, Pilon ${ }^{19}$ was used to perform error correction of p-contigs, and FragScaff ${ }^{37}$ was used to generate scaffolds. Finally, the anchorage of the genome assembly onto chromosomes was performed by the LACHESIS pipeline ${ }^{38}$.

To assess the quality of the assemblies (accuracy and completeness), Illumina clean reads were mapped to our assembly using $\mathrm{BWA}^{39}$. In addition, CEGMA (Core Eukaryotic Genes Mapping Approach) ${ }^{20}$, BUSCO (Benchmarking Universal Single-Copy Orthologs, embryophyta_odb10 database ${ }^{40}$, and the LTR Assembly Index (LAI ${ }^{23}$ were used to assess the genome.

\section{Annotation of repetitive elements}

The repetitive elements of both assemblies were identified using combined strategies, homology-based and de novo prediction. RepeatModeler (http://www. repeatmasker.org/RepeatModeler.html), RepeatScout ${ }^{41}$ 
(http://www.repeatmasker.org/), and LTR-Finder ${ }^{42}$ (http://tlife.fudan.edu.cn/ltr_finder) were applied to ab initio repeat element library construction with default parameters, and RepeatProteinMask and RepeatMasker (http://www.repeatmasker.org) were used to identify repeats. The homologous annotations of repetitive elements were also performed using RepeatProteinMask and RepeatMasker (http://www.repeatmasker.org) by alignment to the consensus sequences in the Repbase library $^{43}$.

\section{Prediction and functional annotation of genes}

Combined strategies, including ab initio, homologybased, and transcript-assisted strategies, were applied to identify genes in the Coptis chinensis assemblies. EvidenceModeler $^{44}$ (EVM, http://evidencemodeler. sourceforge.net) was applied to integrate the annotation results of all the methods to produce the final gene models. The software PASA ${ }^{45}$ (https://github.com/PASApipeline/ PASApipeline/wiki) was then applied for the identification of alternative splicing variations and untranslated sequences (UTRs) of genes. The domains and motifs of the gene models were identified by InterProScan ${ }^{46}$. Gene function annotations were performed by aligning them to the InterPro, NR, SwissProt, and KEGG databases.

\section{Synteny analyses}

All genes were compared in pairs by BLASTP ( $E$ value $<$ $10^{-5}$ ) to obtain orthologous gene pairs. Collinear blocks containing fewer than five orthologous gene pairs were filtered out by MCScanX ${ }^{47}$. The collinearity information of the gene set and other genomic features in the $C$. chinensis genome were visualized by Circos (v0.69) ${ }^{48}$.

\section{Gene family clustering}

The protein sequences of Coptis chinensis and 7 other plant species (Aquilegia coerulea, Arabidopsis thaliana, Artemisia annua, Glycyrrhiza uralensis, Macleaya cordata, Nelumbo nucifera, and Salvia miltiorrhiza) with the outgroup species Oryza sativa were used for gene family clustering. Genes encoding no more than 50 amino acids were removed. For all the genes of the nine species, when there were multiple transcripts in the same gene, only the longest was used for subsequent analysis. The similarity among the remaining genes from all species was obtained by comparing pairs using BLASTP with a cutoff $E$ value of $1 \mathrm{e}-5$. OrthoMCL (http://orthomcl.org/orthomcl/) was used to identify paralogous and orthologous protein sequences among the nine species with an inflation parameter of 1.5.

\section{Phylogenetic analysis}

The coding sequences (CDSs) were extracted from single-copy gene families shared by nine species and were aligned by MUSCLE according to the alignments of the protein sequences. Each family was concatenated into a superalignment matrix. RAxML (http://sco.h-its.org/ exelixis/web/software/raxml/index.html) was applied to construct the maximum likelihood phylogeny using the GTRGAMMA model with Oryza sativa as an outgroup, and the bootstrap value was set to 100 .

\section{Estimation of divergence time}

The MCMCtree program of PAML (http://abacus.gene. ucl.ac.uk/software/paml.html) was implemented to estimate the differentiation time according to the maximum likelihood phylogeny tree with the following settings: 100,000 iterations were discarded, sampling number $=$ 100,000 , and sampling frequency $=2$. The times of divergence between monocotyledons and dicotyledons (148-173 Mya) Aquilegia coerulea and Macleaya cordata (103-122 Mya), Glycyrrhiza uralensis and Arabidopsis thaliana (97-109 Mya), which refer to the TimeTree database (http://www.timetree.org/) and previous stu$\operatorname{dies}^{25,49}$, were used for recalibration.

\section{Gene family analysis}

The gene families of each species that had undergone expansion or contraction were identified by comparison with the number of genes contained in each ancestral gene family using CAFE software (version 1.6). All gene family changes along each lineage of the phylogenetic tree were studied by the random birth and death model. The extrapolation of changes in the gene family size and the direction of changes in the phylogeny were ascertained by the probabilistic graphical model.

\section{Positive selection gene}

The protein sequences of single-copy gene families from Coptis chinensis, Arabidopsis thaliana, Aquilegia coerulea, Glycyrrhiza uralensis, Salvia miltiorrhiza, Artemisia annua, Macleaya cordata, and Nelumbo nucifera were extracted and aligned by MUSCLE (version 3.7). Then, Gblocks (version 0.91b85) was used to extract the conserved sites of the protein sequence alignments. The Codeml program of PAML software (version 4.7) was applied to estimate the evolutionary rate of each branch with a free-ratio model. Then, we detected the positive selection signals on genes of each family using the branchsite model with $C$. chinensis as the foreground branch and the other seven species as the background branch. The likelihood ratio test was used to detect candidates that underwent positive selection with a cutoff $p$ value of 0.05 .

\section{Whole-genome duplication analysis}

The protein sequences from the Coptis chinensis, Aquilegia coerulea, Macleaya cordata, and Nelumbo nucifera genomes were used to analyze WGD events. The BLASTP program of blastall (version 2.2.26) was used to 
perform a homolog search with a cutoff $E$ value of $10^{-5}$, and MCScanX was applied to obtain syntenic blocks with default parameters. Finally, the gene pairs located in syntenic blocks were aligned using MUSCLE, and Ks (synonymous substitution rate) values were calculated using the YN algorithm to detect putative WGD events ${ }^{50}$.

\section{High-performance liquid chromatography analysis}

Dry powder samples $(0.2 \mathrm{~g})$ were extracted with $50 \mathrm{ml}$ hydrochloric acid ethanol mixed liquor $(1: 100, \mathrm{v} / \mathrm{v})$ for $30 \mathrm{~min}$ and sonicated for $30 \mathrm{~min}$. Determination of the major bioactive components of C. chinensis was performed on an Agilent 1260 HPLC system (Agilent Technologies, Santa Clara, Calcium, USA) with an Agilent ZORBAX SB-C 18 column $(250 \mathrm{~mm} \times 4.6 \mathrm{~mm}, 5 \mathrm{~mm}$, Agilent Technologies) at a column temperature of $30^{\circ} \mathrm{C}$ and a flow rate of $1 \mathrm{~mL} / \mathrm{min}$. The mobile phase was acetonitrile $-0.05 \mathrm{~mol} / \mathrm{L}$ potassium dihydrogen phosphate solution $(50: 50, \mathrm{v} / \mathrm{v})$, containing $0.1 \%$ sodium dodecyl sulfate, separated by equal-diameter elution. The detection wavelength was $345 \mathrm{~nm}$. The contents of berberine, palmatine, cannine, coptidine, magnoline, and columbine were calculated by standard curves ${ }^{28,51}$. One-way analysis of variance was used to compare various BIA contents among roots in different years. If the variances were significantly different, Tamhane's T2 test was used to perform post hoc analyses; otherwise, a least significant difference test was used. The results were considered significant at $p<0.05$.

\section{Accession numbers}

All raw and processed sequencing data generated in this study have been uploaded to the NCBI BioProject database under accession number PRJNA649082. The raw genome sequencing data obtained by the Illumina and PacBio platforms were uploaded to the NCBI BioSample database under accession number SAMN15658056. The raw sequencing data of the transcriptome have been uploaded to the NCBI BioSample database under accession number SAMN15658057.

\section{Acknowledgements}

We are grateful for the Illumina sequencing services provided by Novogene Bioinformatics Technology Co., Ltd. (Beijing, China). This study was supported by the Basic Research Projects of Chongqing Province (No. cstc2017jxjl-jbky120013), China National Science and Technology Project of the 12th 5-Year Plan (2011BAl13B02-1) and Chongqing Platform and Base Construction Project (cstc2014ptjd10001).

\footnotetext{
Author details

${ }^{1}$ Chongqing Academy of Chinese Materia Medica, 400065 Chongqing, China. ${ }^{2}$ Chongqing Engineering Research Center for Fine Variety Breeding Techniques of Chinese Materia Medica, 400065 Chongqing, China. ${ }^{3}$ Chongqing Sub-center of National Resource Center for Chinese Materia Medica, China Academy of Chinese Medical Science, 400065 Chongqing, China. ${ }^{4}$ Novogene Bioinformatics Institute, Building 301, Zone A10 Jiuxianqiao North 13 Road, Chaoyang District, 100083 Beijing, China
}

\section{Author contributions}

D.X.C. and L.Y.L. designed the project; D.X.C., Y.P., and Y.W. collected samples; Y Z.C. and Y.J.Z., T.L.S. contributed to molecular experiments and bioinformatics analysis; X.D.H., D.X.C., and Y.P. wrote the paper; R.Y.M., X.L.W., J.T., J.Z., L.A.G., and $X . Z$. revised the paper. All authors have read and approved the final version of the paper.

\section{Conflict of interest}

The author declares no competing interests.

Supplementary information The online version contains supplementary material available at https://doi.org/10.1038/s41438-021-00559-2.

Received: 9 December 2020 Revised: 3 March 2021 Accepted: 14 March 2021

Published online: 01 June 2021

\section{References}

1. Li, X. K., Motwani, M., Tong, W., Bornmann, W. \& Schwartz, G. K. Huanglian, A chinese herbal extract, inhibits cell growth by suppressing the expression of cyclin B1 and inhibiting CDC2 kinase activity in human cancer cells. Mol. Pharmacol. 58, 1287 (2000).

2. Franzblau, S. G. \& Cross, C. Comparative in vitro antimicrobial activity of Chinese medicinal herbs. J. Ethnopharmacol. 15, 279-288 (1986).

3. Liu, B., Li, W., Chang, Y., Dong, W. \& Ni, L. Extraction of berberine from rhizome of Coptis chinensis Franch using supercritical fluid extraction. J. Pharm. Biomed. Anal. 41, 1056-1060 (2006).

4. Lin, C. C., Ng, L. T., Hsu, F. F., Shieh, D. E. \& Chiang, L. C. Cytotoxic effects of Coptis chinensis and Epimedium sagittatum extracts and their major constituents (berberine, coptisine and icariin) on hepatoma and leukaemia cell growth. Clin. Exp. Pharmacol. Physiol. 31, 65-69 (2004).

5. Yan, D., Jin, C., Xiao, X. H. \& Dong, X. P. Antimicrobial properties of berberines alkaloids in Coptis chinensis Franch by microcalorimetry. J. Biochem. Biophys. Methods 70, 845-849 (2008).

6. Diogo, C. V. et al. Berberine as a promising safe anti-cancer agent-is there a role for mitochondria? Curr. Drug Targets 12, 850-859 (2011).

7. Song, X., Pan, Y., Li, L., Wu, X. \& Wang, Y. Composition and diversity of rhizosphere fungal community in Coptis chinensis Franch. continuous cropping fields. PLoS ONE 13, e0193811 (2018).

8. Yan, $\mathrm{H}$. et al. Anti-ultraviolet radiation effects of Coptis chinensis and Phellodendron amurense glycans by immunomodulating and inhibiting oxidative injury. Int. J. Biol. Macromol. 48, 720-725 (2011).

9. Chang, W. Non-coding RNAs and berberine: a new mechanism of its antidiabetic activities. Eur. J. Pharmacol. 795, 8-12 (2017).

10. Yang, T. C. et al. Alkaloids from Coptis chinensis root promote glucose uptake in C2C12 myotubes. Fitoterapia 93, 239-244 (2014).

11. Liu, Y. M. et al. Berberine attenuates depressive-like behaviors by suppressing neuro-inflammation in stressed mice. Brain Res. Bull. 134, 220-227 (2017).

12. Wang, L. et al. New enantiomeric isoquinoline alkaloids from coptis chinensis. Phytochemistry Lett. 7, 89-92 (2014).

13. Chen, H. B. et al. Anti-inflammatory activity of coptisine free base in mice through inhibition of NF-KB and MAPK signaling pathways. Eur. J. Pharmacol. 811, 222-231 (2017).

14. Wu, J. et al. Coptisine from Coptis chinensis inhibits production of inflammatory mediators in lipopolysaccharide-stimulated RAW 264.7 murine macrophage cells. Eur. J. Pharmacol. 780, 106-114 (2016).

15. Wang, D., Liu, Z., Guo, M. \& Liu, S. Structural elucidation and identification of alkaloids in Rhizoma Coptidis by electrospray ionization tandem mass spectrometry. J. Mass Spectrom. 39, 1356 (2004).

16. Xie, W., Gu, D., Li, J., Cui, K. \& Zhang, Y. Effects and action mechanisms of berberine and rhizoma coptidis on gut microbes and obesity in high-fat dietfed C57BL/6J mice. Plos ONE 6, e24520 (2011).

17. Dong, $X$. et al. De novo assembly of a wild pear (Pyrus betuleafolia) genome. Plant Biotechnol. J. 18, 581-595 (2020).

18. Huang, $X$. et al. Genome-wide association studies of 14 agronomic traits in rice landraces. Nat. Genet. 42, 961-967 (2010). 
19. Walker, B. J. et al. Pilon: an integrated tool for comprehensive microbial variant detection and genome assembly improvement. PLOS ONE 9, e112963 (2014).

20. Parra, G., Bradnam, K. \& Korf, I. CEGMA: a pipeline to accurately annotate core genes in eukaryotic genomes. Bioinformatics 23, 1061-1067 (2007).

21. Mochamad, A. F. et al. KNApSAcK family databases: integrated metabolite-plant species databases for multifaceted plant research. Plant Cell Physiol. 53, e1 (2012).

22. Pei, L. et al. Genome and transcriptome of Papaver somniferum Chinese landrace CHM indicates that massive genome expansion contributes to high benzylisoquinoline alkaloid biosynthesis. Hortic. Res. 8, 5 (2021).

23. Ou, S., Chen, J. \& Jiang, N. Assessing genome assembly quality using the LTR Assembly Index (LAI). Nucleic Acids Res. 46, e126 (2018).

24. Li, L., Stoeckert, C. J. Jr \& Roos, D. S. OrthoMCL: identification of ortholog groups for eukaryotic genomes. Genome Res .13, 2178-2189 (2003).

25. Liu, X. et al. The genome of medicinal plant macleaya cordata provides new insights into benzylisoquinoline alkaloids metabolism. Mol. Plant 10, 975-989 (2017).

26. Paterson, A. H. et al. Repeated polyploidization of Gossypium genomes and the evolution of spinnable cotton fibres. Nature 492, 423-427 (2012).

27. Filiault, D. L. et al. The Aquilegia genome provides insight into adaptive radiation and reveals an extraordinarily polymorphic chromosome with a unique history. Elife 7, https://doi.org/10.7554/eLife.36426 (2018).

28. He, S. M. et al. Identification and characterization of genes involved in benzylisoquinoline alkaloid biosynthesis in Coptis species. Front. Plant Sci. 9, 731 (2018).

29. Xie, J. et al. A chromosome-scale reference genome of Aquilegia oxysepala var. kansuensis. Hortic. Res. 7, 113 (2020).

30. Sun, Q., He, X. H., Mu, J. \& Cai, Y. F. Genome survey sequencing and analyzing of Coptis chinensis based on next-generation sequencing. J. Sichuan Univ. (Nat. Sci. Ed.) 51, 1330-1334 (2014).

31. Meyers, B. C., Tingey, S. V. \& Morgante M. Abundance, distribution, and transcriptional activity of repetitive elements in the maize genome. Genome Res. 11, 1660-1676 (2001)

32. Ming, R., Vanburen, R., Wai, C. M., Tang, H. \& Yu, Q. The pineapple genome and the evolution of CAM photosynthesis. Nat. Genet. 47, 1435-1442 (2015).

33. Xia, E.-H. et al. The tea tree genome provides insights into tea flavor and independent evolution of caffeine biosynthesis. Mol. Plant 10, 866-877 (2017).

34. Marçais, G. \& Kingsford, C. A fast, lock-free approach for efficient parallel counting of occurrences of k-mers. Bioinformatics 27, 764-770 (2011).
35. Chin, C. S. et al. Phased diploid genome assembly with single-molecule realtime sequencing. Nat. Methods 13, 1050-1054 (2016).

36. Chin, C. S. et al. Nonhybrid, finished microbial genome assemblies from longread SMRT sequencing data. Nat. Methods 10, 563-569 (2013).

37. Mostovoy, Y. et al. A hybrid approach for de novo human genome sequence assembly and phasing. Nat. Methods 13, 587-590 (2016)

38. Yaffe, E. \& Tanay, A. Probabilistic modeling of Hi-C contact maps eliminates systematic biases to characterize global chromosomal architecture. Nat. Genet. 43, 1059-1065 (2011).

39. Li, H. \& Durbin, R. Fast and accurate short read alignment with BurrowsWheeler transform. Bioinformatics 25, 1754-1760 (2009).

40. Simão, F. A., Waterhouse, R. M., loannidis, P., Kriventseva, E. V. \& Zdobnov, E. M. BUSCO: assessing genome assembly and annotation completeness with single-copy orthologs. Bioinformatics 31, 3210-3212 (2015).

41. Price, A. L., Jones, N. C. \& Pevzner, P. A. De novo identification of repeat families in large genomes. Bioinformatics 21(Suppl 1), i351-358 (2005).

42. Xu, Z. \& Wang, H. LTR_FINDER: an efficient tool for the prediction of full-length LTR retrotransposons. Nucleic Acids Res. 35, W265-268 (2007).

43. Jurka, J. et al. Repbase Update, a database of eukaryotic repetitive elements. Cytogenet. Genome Res. 110, 462-467 (2005).

44. Haas, B. J. et al. Automated eukaryotic gene structure annotation using EVidenceModeler and the program to Assemble Spliced Alignments. Genome Biol. 9, R7 (2008).

45. Haas, B. J. et al. Improving the Arabidopsis genome annotation using maximal transcript alignment assemblies. Nucleic Acids Res. 31, 5654-5666 (2003).

46. Zdobnov, E. M. \& Apweiler, R. InterProScan-an integration platform for the signature-recognition methods in InterPro. Bioinformatics 17, 847-848 (2001).

47. Wang, Y. et al. MCScanX: a toolkit for detection and evolutionary analysis of gene synteny and collinearity. Nucleic Acids Res. 40, e49 (2012).

48. Krzywinski, M. et al. Circos: an information aesthetic for comparative genomics. Genome Res. 19, 1639-1645 (2009).

49. Guo, L. et al. The opium poppy genome and morphinan production. Science 362, 343-347 (2018).

50. Yang, Z. \& Nielsen, R. Estimating synonymous and nonsynonymous substitution rates under realistic evolutionary models. Mol. Biol. Evol. 17, 32-43 (2000).

51. He, S. M. et al. Identification of candidate genes involved in isoquinoline alkaloids biosynthesis in Dactylicapnos scandens by transcriptome analysis. Sci. Rep. 7, 9119 (2017). 